\title{
Downregulation of 15-lipoxygenase 2 by glucocorticoid receptor in prostate cancer cells
}

\author{
YING FENG $^{1}$, XIANGYANG BAI ${ }^{1}$, QIUHUI YANG ${ }^{1}$, HONG WU $^{2}$ and DIAN WANG ${ }^{1}$ \\ Departments of ${ }^{1}$ Radiation Oncology, ${ }^{2}$ Physical Medicine and Rehabilitation, Medical \\ College of Wisconsin, 8701 Watertown Plank Rd, Milwaukee, WI 53226, USA
}

Received December 22, 2009; Accepted February 22, 2010

DOI: 10.3892/ijo_00000641

\begin{abstract}
LOX-2) is lost or significantly reduced in prostate cancer. However, the regulation of 15-LOX-2 remains unclear. In this study, we independently cloned the 5' upstream promoter fragments of 15-LOX-2 gene. Target DNA fragments each were cloned into an expression vector containing luciferase reporter gene, which were called LF1(-1533/+87), LF2(-628/+87), LF3(-253/+87), LF4(-157/ +87), LF5(-33/+87), LF6(-253/+1), and LF7(-157/+1). Each of these individual promoter fragments was transfected into primary prostate epithelial cells and prostate cancer LNCaP cells. The promoter activity gradually decreased with progressive deletions from LF2 to LF4. A significant drop was noted in the LF5. LF6 and LF7 that did not contain the 87-bp region downstream the transcription start site (TSS) have significant luciferase activities similar to those of corresponding fragments (LF3 and LF4) that contain 87-bp region downstream the TSS. This suggests that the 125-bp region $(-157$ to -33$)$ of $L F 4$ is critical for the promoter activity of $15-\mathrm{LOX}-2$ in the primary prostate epithelial cells PrEC and cancer cells LNCaP. Moreover, we discovered a specific glucocorticoid receptor (GR) responsive element (GRE) in this key region. The luciferase activities of the LF4 and LF7 were decreased in the LNCaP cells co-transfected with GR (hGR $\alpha$ or hGRß) expression vectors. This inhibitory effect is reversed after treatments with dexamethasone or two specific GR inhibitors (siRNAs of GR and RU486). Results from this study suggest a 125 -bp region $(-157$ to -33$)$ is critical for the 15-LOX-2 promoter activity in prostate epithelial cells and
\end{abstract}

Correspondence to: Dr Dian Wang, Department of Radiation Oncology, Medical College of Wisconsin, 9200 West Wisconsin Ave, Milwaukee, WI 53226, USA

E-mail: dwang@mcw.edu

Abbreviations: COX, cyclooxygenase; EMSA, electrophoretic mobility shift assay; GR, glucocorticoid receptor; HRPC, hormone refractory prostate cancer; 15-LOX-2, 15-lipoxygenase 2; PC, prostate cancer

Key words: 15-lipoxygenase 2, glucocorticoid receptor, prostate cancer, promoter cancer cells, which was significantly downregulated by GR via the GRE in this region.

\section{Introduction}

15-lipoxygenase 2 (15-LOX-2) is a 76-kDa enzyme and consists of 662 amino acids and two domains (www.ncbi.nlm. nih.gov). The domain one called Plat_LOX domain contains 110 amino acids located in residual number 2 through 111. The domain two called lipoxygenase domain contains 518 amino acids located in residual number 137 through 654. 15LOX-2 oxygenates carbon-15 in arachidonic acid (AA) and is one of important lipid peroxidizing enzymes that have been linked to carcinogenesis including prostate cancer. Unlike 15-lipoxygenase 1 (15-LOX-1), 15-LOX-2 has limited tissue distribution and significant substrate preference (1). 15-LOX-2 is mainly expressed in epithelia from prostate (2), esophagus (3) 3, head and neck (4), breast, bladder, lung (5), and skin $(1,5)$. 15-LOX-2 preferentially converts arachidonic acid to 15-S-hydroxyeicosatetraenoic acid (15-S-HETE) and metabolizes linoleic acid poorly.

15-LOX-2 has been considered to be a tumor suppressor. First, 15-LOX-2 is significantly reduced or lost in cancer of prostate (2), esophagus (3), head and neck (3) breast, bladder, lung, and skin (5). Second, immunohistochemical studies further showed that the reduced expression of 15-LOX-2 was inversely correlated with the degree of tumor differentiation and pathologic grade (Gleason scores) in prostate cancer (6). 15-LOX-2 expression was decreased in high-grade prostatic intraepithelial neoplasm (HGPIN) compared with benign tissues, suggesting that the loss of $15-\mathrm{LOX}-2$ is an early event in prostate carcinogenesis (6). Third, results from us (4) and others $(2,3)$ showed that restoration of $15-\mathrm{LOX}-2$ resulted in significant inhibition of cancer growth proliferation. Moreover, 15-LOX-2 is found to be a negative cell cycle regulator in normal prostate epithelial cells (7) and may be associated with the ability to induce cell senescence (8). All together, 15-LOX-2 plays an anti-carcinogenic role in many epitheliaderived carcinomas including prostate cancer.

It remains unclear why tumor suppressor 15-LOX-2 is lost or significantly reduced in epithelia-derived cancer including prostate cancer. The previous study (7) revealed that loss of 15-LOX-2 expression in prostate cancer cells is not caused by promoter (gene) hypermethylation or histone 
deacetylation. Recently there were two other studies focused on the promoter regulation of 15-LOX-2 gene. Tang et al (9) reported that $\mathrm{Sp} 1$ positively and $\mathrm{Sp} 3$ negatively regulate and androgen does not regulate $15-\mathrm{LOX}-2$ gene expression in normal human prostate epithelial cells. Subbarayan et al (5) reported that $15-\mathrm{LOX}-2$ can be negatively regulated by its product 15-(S)-hydroxyeicosatetraenoic acid and PPAR $\gamma$ through a PPAR half-site present in the 15-LOX-2 promoter region (-560 to -596$)$. The above studies might partly explain the gene regulation of $15-\mathrm{LOX}-2$ in prostate cancer, but additional studies are clearly required to reveal molecular mechanisms for the gene regulation of this functional tumor suppressor 15-LOX-2 in prostate cancer. This information would be important leading to the identification of a molecular target(s) for prevention, early diagnosis and treatment of prostate cancer.

In this study, we independently cloned 5' upstream promoter fragments of the human 15-LOX-2 gene from human fetal skin DNA through progressive deletions. Sequences of these fragments were verified. These promoter fragments each were cloned into the expression vector containing luciferase reporter gene. The luciferase activities of these promoter fragments each were examined in the primary normal prostate epithelial cells and the prostate cancer cell line LNCaP. We determined a 125 -bp fragment (-157 to -33) as a key region for the promoter activity of $15-\mathrm{LOX}-2$ and identified a specific glucocorticoid receptor (GR) responsive element (GRE) in this key region through a computer search analysis and a gel shifting experiment. Furthermore, we have determined that 15 -LOX-2 promoter was downregulated by overexpression of GR in prostate cancer cells. This inhibitory effect by GR was reversed when the cells were treated with either steroid dexamethasone, or a specific GR inhibitor (siRNA of GR or RU486).

\section{Materials and methods}

Cloning of immediate upstream 15-LOX2 promoter region. Primer design: the human 15-LOX-2 gene was found to be located in chromosome 17 (10). The primer sequences (Table I) for cloning were designed based on the genomic sequences and cDNA sequence of 15-LOX-2 deposited in the GenBank (Accession numbers: NT_086857, AJ305028-AJ305031).

Cloning of 15-LOX2: Fetal skin genomic DNA was purchased from the American Type Culture Collection (ATCC, Manassas, VA). Expand long template PCR system and Tag DNA polymerases were obtained from Roche Diagnostics Corporation, Roche Applied Science (Indianapolis, IN). TA cloning ${ }^{\circledR}$ kit was purchased from Invitrogen (Carlsbad, CA). The PCR for target DNA fragments was performed using Bio-Rad icycler (Bio-Rad, Hercules, CA). These PCR products were gel purified using QIAquick ${ }^{\circledR}$ gel extraction kit (Qiagen, Valencia, CA), and cloned respectively into the T/A vector pCRII and transformed into OneShot cells (Invitrogen) following the manufacturer's protocol. These cells were cultured in LB medium containing $100 \mu \mathrm{g} / \mathrm{ml}$ ampicillin. Plasmids were purified with Concert ${ }^{\mathrm{TM}}$ Rapid Plasmid Miniprep System (Gibco-BRL, Rockville, MD). The target DNA fragments were further excised, gel purified and cloned into pGAW expression vector (gifted from Dr Fei Xiao, University of Stanford, CA). The orientation and sequence of each insert were confirmed by restriction digestion and sequencing. A dye terminator cycle sequencing was performed to determine sequence of each of DNA fragments using an ABIPRISM $^{\mathrm{TM}} 310$ Genet Analyzer. The other primers that were used for PCR and sequencing, were T7, SP6 primers, pGAW-For-primer, luc-Rev-primer designed according to the sequence of the parental pGAW vector (Table I). A computer analysis was performed on the nucleotide sequence of each clone using the software from the National Center for Biotechnology Information BLAST network service.

Cell cultures. The prostate cancer cell line LNCaP was obtained from ATCC. The LNCaP cells were cultured in RPMI-1640 medium supplemented with $10 \%$ heat-inactivated fetal bovine serum (FBS) plus $1 \mathrm{X}$ antibiotic-antimycotic solutions (Gibco, Grand Island, NY). A normal primary prostate epithelial cell line PrEC was purchased from Clonetics and maintained in PrEBM media (Cambrex or Clonetics, Charles City, IA). Cultures were maintained in a humidified incubator with $5 \% \mathrm{CO}_{2}, 95 \% \mathrm{O} 2$ air at $37^{\circ} \mathrm{C}$, and the media was changed twice a week.

Luciferase reporter assays. Luciferase reporter assays were performed in the cells. The cells grown in six-well culture plates $\left(5 \times 10^{5}\right.$ cells/well $)$ were transiently transfected, in triplicate, with either promoterless parental vector pGAW or various luc constructs ( $2 \mu \mathrm{g}$ plasmid/well) together with the B-gal plasmid $(0.125 \mu \mathrm{g}$ plasmid/well) to normalize the transfection efficiency. To determine effect of human glucocorticoid receipt (GR) on promoter activities of 15-LOX-2, a LF fragment was co-transfected with a GR expression vector into the LNCaP cells. The human GR expression vectors (pcDNA3-hGR $\alpha$ containing $\alpha$ isoform of GR and pcDNA3hGRß containing $\beta$ isoform of GR) were kindly provided by Dr Julian R.E. Davis, Manchester University, UK. Forty eight hours after the transfection, the cells were harvested by scraping into $1 \mathrm{X}$ lysis buffer (Promega, Madison, WI) for a subsequent measurement of luciferase activity.

Lysates containing equal amounts of protein were assayed for luciferase activity using luciferase assay kit (Promega). ß-galactosidase activity was measured using Tropix GalactoLight Plus assay system. After subtracting the baseline values, the relative luciferase unit (RLU) activity was determined as the ratio of luciferase/ß-gal activities. The relative promoter activities were expressed as relative values (i.e. \%).

Electrophoretic mobility supershift assays (EMSA). Immunoprecipitation (IP) of GR protein was performed using LANCaP cells. Briefly the cells were transfected with pCDNA3.1-hGR $\alpha$. After the selection in the media containing G418 for 2 weeks, the surviving tumor cells were collected and the nuclear lysates were generated using a lysis buffer $(50 \mathrm{mM}$ Tris- $\mathrm{HCl}, \mathrm{pH} 8.0$, $150 \mathrm{mM} \mathrm{NaCl}, 1 \% \mathrm{NP}-40)$. The nuclear lysates were then incubated overnight at $4^{\circ} \mathrm{C}$ with Sepharose-G bind beads (Santa Cruz Biotechnology, Inc., Santa Cruz, CA) to which rabbit anti-GR antibody (Santa Cruz Biotechnology, Inc) was bound. These beads were then washed, collected and subjected to EMSA on $6 \%$ polyacrylamide gels.

One 26-bp double-stranded oligonucleotide probe containing putative GR binding element and a double-stranded oligonucleotide probe containing mutations in the core of 
Table I. Primers and oligonucleotides for cloning, sequencing, electrophoretic mobility supershift assay (EMSA) and sitedirected mutagenesis (putative and mutant GRE are highlighted).

Name

Sequence

Restricted

enzyme

Cloning primers
LOX2-1R
LOX2-2F
LOX2-3F
LOX2-4F
LOX2-5F
LOX2-6F
LOX2-7F
LOX2-8R
LOX2-9F
LOX2-10F

Sequencing primers

pGAW-For-primer

Luc-Rev-primer

Gel shift oligonucleotides

GRGS-1

GRGS-2

GRGSM-3

GRGSM-4

Primers for site-directed mutagenesis GRGSM-5

Amp rev

\author{
5'-ATCGGTACCGCTGCCAGCCTAAGTCCAG-3' \\ 5'-ACTCTCGAGAGGGCACAGCTTGTGCCTC-3' \\ 5'-AGCCTCGAGCGCCTGTAATCCCAGCACT-3' \\ 5'-TAGCTCGAGCACTTGAACCCAGGAGGAG-3' \\ 5'-ATCCTCGAG CAGACGTGGTGGGCACAAA-3' \\ 5'-ACCCTCGAG CCTCAGGGTATTCTTAGTC-3' \\ 5'-ATTCTCGAG GAGGAGTCCACTGGGCTTG-3' \\ 5'-ACATCAGATCTTTATTGCCACTGACTCCAAGC-3' \\ 5'-AGCAAGCTTCAGACGTGGTGGGCACAAA-3' \\ 5'-AGCAAGCTTCCTCAGGGTATTCTTAGTC-3'
}

KpnI

XhoI

XhoI

XhoI

XhoI

XhoI

XhoI

BgIII

HindIII

HindIII
5'-CTAGCAAAATAGGCTGTCCC-3'
5'-TCCAGCGGATAGAATGGCGC-3'

5'-CTTAGTCCCTCCTGTTCCCAGCGCTG-3'

5'-GCTGGGAACAGGAGGGACTAAGAATA-3'

5'-CTTAGTCCCTACTGACCCCAGCGCTG-3'

5'-GCTGGGGTCAGTAGGGA2CTAAGAATA-3'
5'-CTGGGGTCAGTAGGGACTAAGAATACCCTGAG-3'
5'-GTCAGAAGTAAGTTGGCCGCAGTGTTATCACTCATGG-3' putative GR binding element were designed and synthesized (Table I). The probes were designed based on the putative sequence CTCCTGTTCC of GRE, among which the sequence TGTTCC is critically required (11). These probes were labeled with or without biotin using the Biotin 3' End DNA Labeling kit (Pierce, Rockford, IL). The gel shift reaction was accomplished by using the LightShift Chemiluminescent EMSA kit (Pierce) according to the manufacturer's instructions. For each gel shift reaction, an aliquot of labeled oligonucleotide (20 fmoles) was incubated with $3 \mu \mathrm{g}$ of nuclear extract from the cells for $20 \mathrm{~min}$ at room temperature in a $10-\mu 1$ mixture containing $1 \mathrm{X}$ binding buffer, $2.5 \%$ glycerol, $10 \mathrm{mmol} / 1 \mathrm{MgCl}_{2}$, $50 \mathrm{mg} / \mathrm{l}$ poly (deoxyinosinic-deoxycytidylic acid), and $0.05 \%$ Nonidet P-40. Unlabeled probes with either 200- or 400-fold molar excess were used in order to competitively block the binding of GR to labeled probes. The unlabeled probes were first preincubated for $2 \mathrm{~min}$ at room temperature with the GR IP extracts before the addition of the labeled probe. Samples were run on a non-denaturing $6 \%$ polyacrylamide gel in $0.5 \mathrm{X}$ Tris-borate EDTA buffer at $100 \mathrm{~V}$ for $70 \mathrm{~min}$ at $4^{\circ} \mathrm{C}$. The electrophoresed binding reactions were transferred to positively charged nylon membrane in a Mini Trans-Blot Cell (Bio-Rad) at $380 \mathrm{~mA}$ for $40 \mathrm{~min}$, and crosslink was performed for $90 \mathrm{sec}$ in an XL-1500 UV Crosslinker chamber (Fisher Scientific, IL). Detection of biotin-labeled DNA was performed using stabilized streptavidin/horseradish peroxidase conjugate (Pierce) according to the manufacturer's procedures.

Treatment with specific GR inhibitors (siRNAs of GR and $R U 486)$ in LANCaP prostate cancer cells. The LANCaP cells were co-transfected with pcDNA3.1-hGR $\alpha$ containing $\alpha$ isoform of GR, and LF4 or LF7. A specific GR inhibitor $1 \mu \mathrm{mol}$ RU486) was added to the cells immediately after the cotransfection or another specific GR inhibitor, siRNAs of GR at 80 pmols concentration was co-transfected with above plasimds. The parental plasmid pcDNA3.1 was used as a control vector for the comparison. Forty-eight hours later the treated cells were harvested. Luciferase reporter assays and Western blot assay were performed using the cells lysates. RU486 was purchased from Sigma-Aldrich, St Louis, MO. siRNA of GR was purchased from Santa Cruz Biotechnology Inc. siRNAs sequences are: sense strand (A): GGCUUCAG GUAUCUUAUGATT, (B): CCAAGAGCUAUUUGAUGA ATT, (C): CUCCAGUAUUCUUGUCAAATT. A phosphorylated GR antibody was purchased from Cell Signaling Technology (Boston, MA).

Site-directed mutagenesis. Site-directed mutagenesis was generated using Change-IT ${ }^{\mathrm{TM}}$ Multiple Mutation Site Directed Mutagenesis kit following by the manufacturer's instruction. The kit was purchased from USB Corporation (Cleveland, $\mathrm{OH}$, 
USA). The GRGSM-5 and amp rev primers were used for the substitutions (Table I). The mutations in the primer GRGSM-5 were the same as in GRGSM-4 which was used in EMSA. The primer GRGSM-5 phosphorylated at their 5' end was synthesized by Invitrogen. Briefly, $5 \mathrm{nmol}$ of each primer, $50 \mathrm{ng}$ pGAWLF7, $0.8 \mu \mathrm{l}$ Change-IT enzyme were mixed together with $20 \mu \mathrm{l}$ volume. The thermal cycler was programmed as follows: initial denaturation at $95^{\circ} \mathrm{C}$ for $2 \mathrm{~min}$; 30 cycles at $95^{\circ} \mathrm{C}$ for $30 \mathrm{sec}, 55^{\circ} \mathrm{C}$ for $30 \mathrm{sec}$ and $68^{\circ} \mathrm{C}$ for $10 \mathrm{~min}$; post-incubation at $72^{\circ} \mathrm{C}$ for $10 \mathrm{~min}$. Two microliters of the final sample was used to transform competent $E$. coli cells DH5 $\alpha$ (Invitrogen). The purified plasmids were sequenced to confirm the mutant substitution successfully. The luciferase activity assay was obtain by transferring mutant LF7 fragment into LNCaP cells with or without Dex or RU486 treatment as above described.

Western blot analysis. The above LNCaP cells were lysed in a buffer containing protease inhibitors. The protein concentration was determined using a bicinchoninic acid (BCA) assay kit (Pierce). Bovine serum albumin was used as a standard. SDS-PAGE was carried out using Bio-Rad gel system. Equal aliquots of total cell lysates (50 $\mu \mathrm{g}$ per sample) were loaded per lane in Laemmli SDS-PAGE sample loading buffer under denaturing conditions. The Western blot was carried out according to the protocol of Santa Cruz Biotechnology, Inc. GAPDH from Santa Cruz Biotechnology was used as an internal control. The blots were quantitatively analyzed using Kodak Electrophoresis Documentation and Analysis System (EDAS) 290 (Eastman KODAK Company, Rochester, NY). Statistical analysis was done by paired Student's t-test and $\mathrm{P}$-values were determined to evaluate the statistical significance $(\mathrm{P}<0.05)$ of the changes observed.

\section{Results}

Characteristics of 5' upper stream structure of 15-LOX-2. A total of 7 fragments were amplified from 5 ' untranslated structure of 15-LOX-2 gene and were cloned through progressive deletions using human fetal skin DNA as a template. These seven fragments were LF1 (-1533/+87, 1620 bp), LF2 (-628/+87, 715 bp), LF3 (-253/+87, 340 bp), LF4 (-157/+87, $244 \mathrm{bp})$, LF5 (-33/+87, $120 \mathrm{bp})$, LF6 (-253/+1, $254 \mathrm{bp})$, and LF7 $(-157 /+1,158$ bp), respectively (Fig. 1A). The LF6 and LF7 are the same to the LF3 and LF4, respectively; except that the 87-bp sequence downstream the transcription start site (TSS) is not included in LF6 and LF7 [the TSS was previously determined (9)]. The sequence analyses were verified using two different amplified fragments from two different human fetal skin DNA samples.

Luciferase activities of the promoter fragments of 15-LOX-2 gene in the prostate cancer cell line LNCaP. Here we evaluated the basal activities of all seven promoter fragments (LF1LF7) in the LNCaP prostate cancer cell line. The luciferase activity of LF1 was normalized as 100 . The luciferase activities of LF2, LF3, LF4, LF5, LF6, LF7 were 131.42 \pm 8.07 , $117.22 \pm 5.29,111 \pm 6.48,1.94 \pm 0.07,94.17 \pm 4.98,100.94 \pm 3.94$ respectively (Fig. 2A). The basal activity of the full-length fragment LF1 was lower than that of LF2. This may suggest that an inhibitory sequence(s) occurs in the LF1 region from

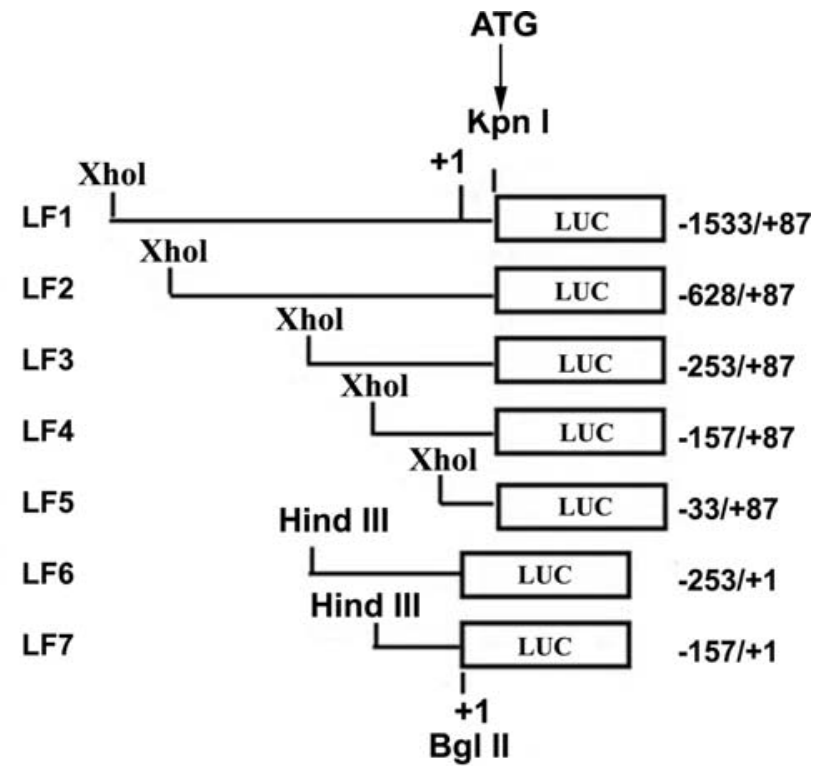

Figure 1. Characteristics and progressive dissection of 5' upstream structure of 15-LOX-2 gene. A, schematic review of dissected fragments, LF1 (-1533/ -87), LF2 (-628/+87), LF3 (-253/+87), LF4 (-157/+87), LF5 (-33/+87), LF6 $(-253 /+1)$, and LF7 $(-157 /+1)$.

-1533 to $-628 \mathrm{bp}$. The promoter activity gradually decreased with progressive deletions from LF2 to LF5. A significant drop was noted in the luciferase activity $(1.94 \pm 0.07)$ of LF5 from the activity $(111 \pm 6.48)$ of LF4. This suggests that a significant enhancer sequence(s) or responsive element(s) occur in the LF4 region from -157 to $-33 \mathrm{bp}$. We further discovered that the fragments (LF6 and LF7) that do not contain the 87-bp region downstream the transcription start site (TSS) (+1 in Fig. 1A) have significant luciferase activities similar to those of corresponding fragments (LF3 and LF4) that contain 87-bp region downstream of the TSS $(+1)$. Similar results were obtained in primary normal prostate epithelial cells PrEC (Fig. 2B). Results from the above studies suggest that the 125 -bp region $(-157$ to -33$)$ is critical for the promoter activity of 15 -LOX-2 gene in the primary prostate epithelial cells and prostate cancer cells.

Existence of a specific glucocorticoid receptor responsive element (GRE) in the 15-LOX-2 promoter fragment. Based on the above discovery that the 125 -bp region $(-157$ to -33$)$ is critical for promoter activity of $15-\mathrm{LOX}-2$ in the prostate cancer cells, we hypothesized that an important transcription factor response element(s) may exist in this region. We have performed an extensive computer analysis to search for transcription binding elements (www.gene-regulation.com). Five putative binding elements of transcription factors GR, Egr-1, Krox-20, GLI3 and ETF were identified in this region, but not in other regions of $15-\mathrm{LOX}-2$ gene promoter structure. Among these 5 putative binding elements, a putative glucocorticoid receptor (GR) responsive element (GRE) (CCTCCTGTTC -139 to -129$)$ was found to partially match with the known consensus sequence GGTTACAnnnTGTTCT for GRE (11-13). In this study, we chose to investigate whether or not expression of GR regulates $15-\mathrm{LOX}-2$ promoter in the LNCaP prostate cancer cells. This is because glucocorticoid is known to inhibit cell proliferation of prostate cancer 


\section{A}

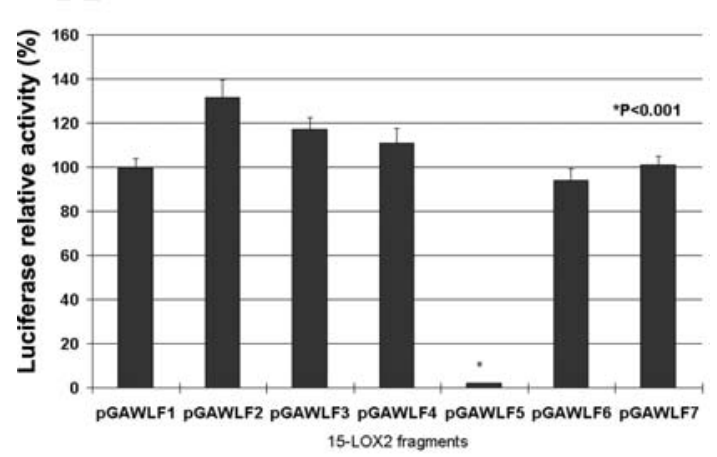

B

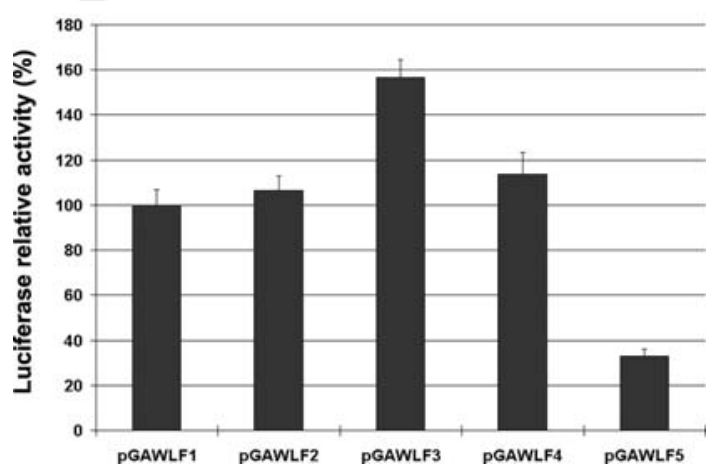

Figure 2. Luciferase acivities of each fragmen in prostate cancer cells LNCaP and in primary prostate epithelial cells (PrEC). (A) Luciferase activities of each fragment in prostate cancer cells LNCaP. The activity of LF1 was normalized as 100. Activities of LF2-7 were $131.42 \pm 8.07,117.22 \pm$ $5.29,111 \pm 6.48,1.94 \pm 0.07,94.17 \pm 4.98,100.94 \pm 3.94$, respectively. (B) Luciferase activities of each fragment in primary prostate epithelial cells (PrEC). The activity of LF1 was normalized as 100. Activities of LF2-7 were $106.41 \pm 6.72,156.67 \pm 7.78,113 \pm 9.41,32.81 \pm 3.26$, respectively. The experiments are expressed in mean $\pm \mathrm{SE}$ and repeated three times.

through its binding to glucocorticoid receptor (GR) (14-17), activation of GR results in the suppression of prostate cancer (18), and GR was not significantly expressed in many prostate cancer cell lines including LNCaP (18).

To examine whether or not this partially matched GRE in the key region of LF4 was indeed bound by GR, we transfected hGR $\alpha$ expression vector into the LANCaP cells. The surviving tumor cells containing hGR $\alpha$ were obtained after the selection with G418. Immunoprecipitation of GR protein were then performed using the nuclear lysates from the tumor cells containing hGR $\alpha$. Finally we performed electrophoretic mobility shift assays (EMSA) to determine the binding of GR to GRE. Results from the EMSA showed that the wild-type double strand oligonucleotide probe containing a putative GR binding element was bound by the immunoprecipated protein containing highly expressed GR (lane 1, Fig. 3). However, this binding was competitively blocked by a high excess of unlabeled probe (Lanes 2 and 3, Fig. 3), but not blocked by the mutant oligonucleotides (lane 4). Fig. 3 is a representative picture. These results indicated that this partially matched GRE is able to be bound by the glucocorticoid receptor.

Downregulation of 15-LOX-2 promoter activity by the glucocorticoid receptor $(G R)$ was reversed by steroid dexamethasone

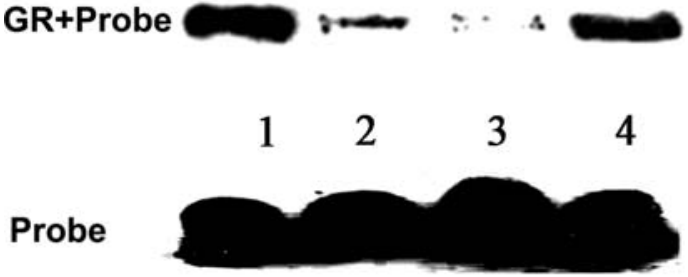

Figure 3. Electrophoretic mobility shift assay for GR binding to 15-LOX-2 promoter. Lane 1, 20 fmoles labeled oligonucleotides; Lane 2, 200-fold molar excess of unlabeled probes before addition of the labeled probe; Lane 3, 400-fold molar excess of unlabeled probes before addition of the labeled probes; Lane 4, 200-fold molar excess of unlabeled mutant probes before the addition of the labeled probes.

(DEX). To address whether or not GR is able to regulate the 15-LOX-2 promoter through the above GRE, we performed functional assays to determine the effect on the lucifease activities of LF4 and LF7 that contain the GRE in the LANCaPs cells co-transfected with a GR (hGR $\alpha$ or hGRß) expression vectors. In addition, we evaluated the effect of dexamethasone (DEX) on the luciferase activities of LF4 and LF7 in the cells co-transfected with a GR expression vector. This is because DEX is known to downregulate GR expression in LANCaP cells $(19,20)$. First, we examined the luciferase activities of LF4 in the LNCaP cells, which was co-transfected with either hGR $\alpha$ or hGRß, with or without dexamethasone (DEX) treatment. LF5 that does not contain the GRE was used as a control. The luciferase activity of LF4 significantly decreased to $59.58 \% \pm 2.74$ when LF4 and hGR $\alpha$ were cotransfected into the cells. However, this downregulation of LF4 activity by hGR $\alpha$ was reversed $(99.56 \% \pm 5.89)$ when the cells were treated with DEX at a concentration of $0.1 \mu \mathrm{M}$ (Fig. 4A). We further evaluated whether or not this effect by DEX was dose-dependent. Interestingly, no significant changes were observed in the luciferase activity of LF4 when much higher concentrations of DEX $(1$ or $2 \mu \mathrm{M})$ were utilized (Fig. 4A). Similarly, the luciferase activity of LF4 was decreased to $68.75 \pm 3.28$ when LF4 and GRß expression vector were co-transfected into the cells. This downregulation of LF4 activity by GRß was slightly reversed by the DEX treatment, but without statistical significance (Fig. 4B). The luciferase activities of LF4 was found to increase slightly (119.72 \pm 3.97 ) with DEX $1 \mu \mathrm{M}$ (Fig. 4E, lane 2), however, the effect by DEX was not dose-dependent since different concentrations of DEX treatment $(0.1 \mu \mathrm{M}$, or 1 or $2 \mu \mathrm{M})$ produced no significant difference in the luciferase activities of the cells co-transfected with LF4 and GRß expression vectors (Fig. 4B). Similar results were obtained when the luciferase activities of LF7 were evaluated in the LNCaP cells that were co-transfected with hGR $\alpha$ or hGR $\beta$ and were treated with or without DEX treatment (Fig. 4C and D, Fig. 5C). LF5 that does not contain the GRE was used as a control. The above results indicate that $15-\mathrm{LOX}-2$ promoter was downregulated by GR, however this inhibitory effect by GR was reversed by steroid DEX treatment.

The inhibitory effect on 15-LOX-2 promoter activity by GR was reversed by a specific GR inhibitor (siRNAs of GR or $R U 486)$. To make sure that the above luciferase activities of 
A

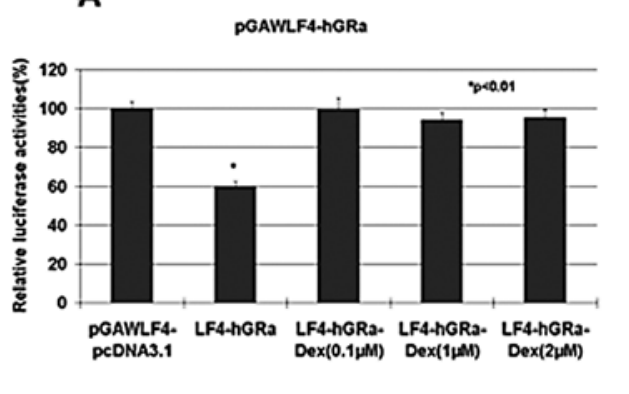

B

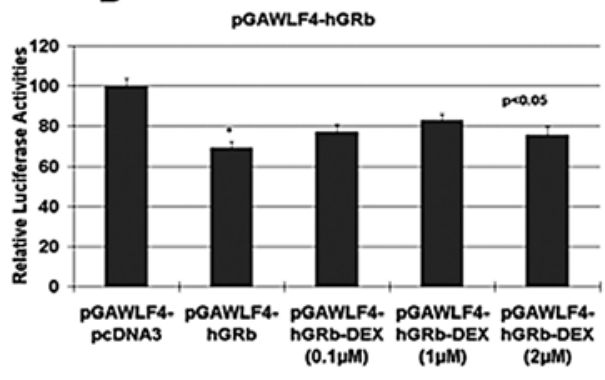

C

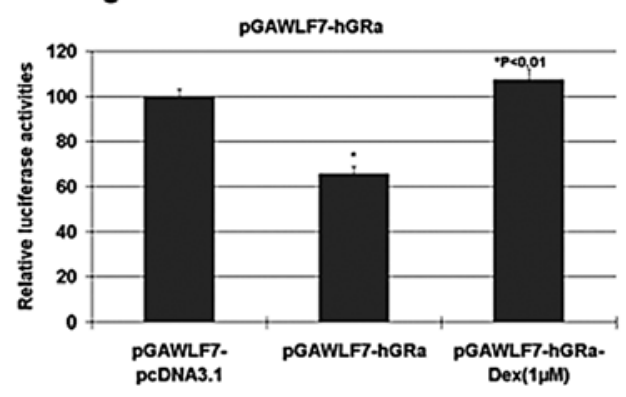

D

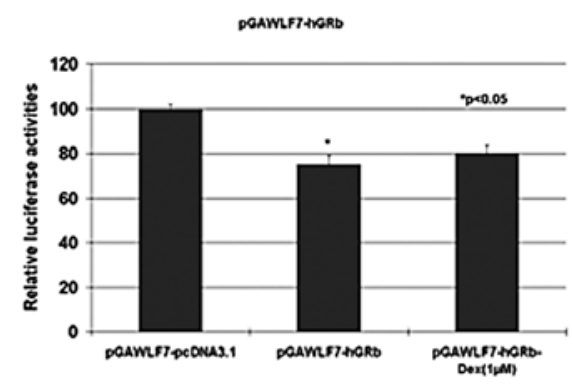

E

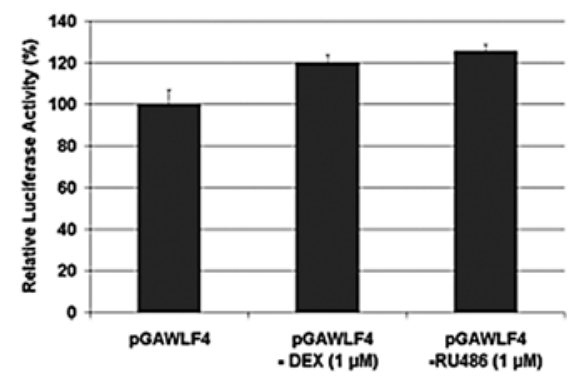

Figure 4. Promoter downregulation of 15-LOX-2 gene by GR and reversion of this GR inhibitory effect by steroid dexamethasone (DEX) in prostate cancer cells LANCaP. Luciferase activities of LF4 or LF7 were measured in the cells co-transfected with/without the GR expression vectors (pcDNA3-hGR $\alpha$ or pcDNA3-hGRß) and at various concentrations of steroid DEX. (A) Co-transfected with pGAWLF4 and parental pcDNA3.1 (activity normalized as 100); Cotransfected with pGAWLF4 and hGR $\alpha$ expression vector (59.58 \pm 2.74$)$; Co-transfected with pGAWLF4 and hGR $\alpha$ expression vector plus DEX (0.1 $\mu \mathrm{M})$ (99.56 \pm 5.89$)$; Co-transfected with pGAWLF4 and hGR $\alpha$ expression vector plus DEX (1 $\mu$ M) (93.91 \pm 3.90$)$; Co-transfected with pGAWLF4 and hGR $\alpha$ expression vector plus DEX $(2 \mu \mathrm{M})(95.15 \pm 4.16)$. (B) Co-transfected with pGAWLF4 and parental pcDNA3.1 (activity normalized as 100); Co-transfected with pGAWLF4 and hGRß expression vector (68.75 \pm 3.28$)$; Co-transfected with pGAWLF4 and hGRß expression vector plus DEX (0.1 $\mu \mathrm{M})(76.99 \pm 3.59)$; Co-transfected with pGAWLF4 and hGRß expression vector plus DEX $(1 \mu \mathrm{M})$ ( (82.94 \pm 3.09$)$; Co-transfected with pGAWLF4 and hGRß expression vector plus DEX $(2 \mu \mathrm{M})(75.29 \pm 4.51)$. (C) Co-transfected with pGAWLF7 and parental pcDNA3.1 (activity normalized as 100); Co-transfected with pGAWLF7 and hGR $\alpha$ expression vector (65.72 \pm 3.22$)$; Co-transfected with pGAWLF7 and hGR $\alpha$ plus DEX (1 $\mu$ M) (107 \pm 4.78$)$. (D) Co-transfected with pGAWLF7 and parental vector pcDNA3.1 (activity normalized as 100); Co-transfected with pGAWLF7 and hGR $\alpha$ expression vector (74.98 \pm 4.05 ); Co-transfected with pGAWLF7 and hGR $\alpha$ expression vector plus DEX $(1 \mu \mathrm{M})(79.62 \pm 4.12)$. (E) pAGWLF4 (activity normalized as 100); pAGWLF4 with DEX (1 $\mu \mathrm{M})$ treatment (119.72 \pm 3.97$)$; pAGWLF4 with RU486 $(1 \mu \mathrm{M})$ treatment (124.25 \pm 3.43$)$.

15-LOX-2 promoter were specifically downregulated by expressed GR, we further evaluated the effect of two specific GR inhibitors (siRNAs of GR and RU486) on the luciferase activities of LF4 and LF7 in the LANCaP cells co-transfected with a GR expression vector compared with controls. The siRNAs of GR was previously shown to specifically abolish expression of GR (21) and RU486 was shown to inhibit the function of 15-LOX-2 $(22,23)$. The luciferase assays showed that the treatment with siRNAs of GR was able to reverse the inhibitory effect of GR on promoter activities of LF4 and LF7, GR reduced luciferase activities of LF4 and LF7 to $60.73 \% \pm 2.09$ and $56.11 \pm 2.94$, respectively, but these were up to $86.17 \pm 3.60$ and $138.57 \pm 3.03$, respectively after the treatment with siRNAs of GR. More effectively, the treatment with RU486 was able to reverse the inhibitory effect of GR on luciferase activities of LF4 from $60.73 \% \pm 2.09$ to
$172 \% \pm 7.78$ and on the activities of LF7 from $56.11 \% \pm 2.94$ to $290.18 \% \pm 9.79$, respectively. The results are summarized in Fig. 5A and B. The inhibitor RU486 slightly increased the luciferase activities of LF4 and LF7 (Fig. 4E, 5C, lane3). In this study, we also performed Western blot analysis of GR in the LANCaP cells, which showed the treatment with the siRNAs of GR completely abolished the GR exogenous or endogenetic expression (Fig. 5D). However, the treatment with RU486 did not affect the expression level of GR (Fig. 5D). This finding was consistent with the previous studies that RU486 block the GR function through high affinity for the glucorticoid receptors, but does not change the expression level of GR (24). The results from the above experiments further confirmed that GR expression downregulated 15LOX-2 promoter through the GRE in the key region of 15-LOX-2. 
A

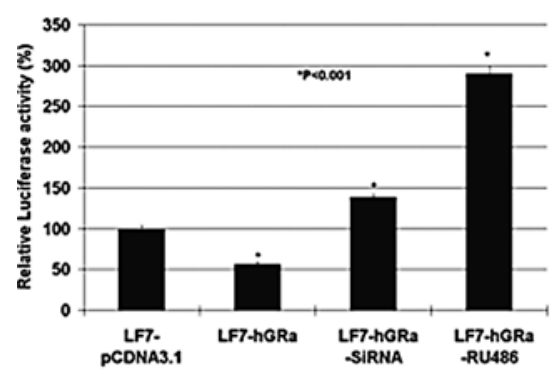

B

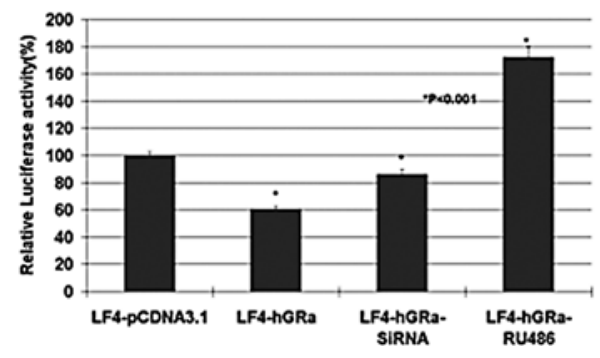

C

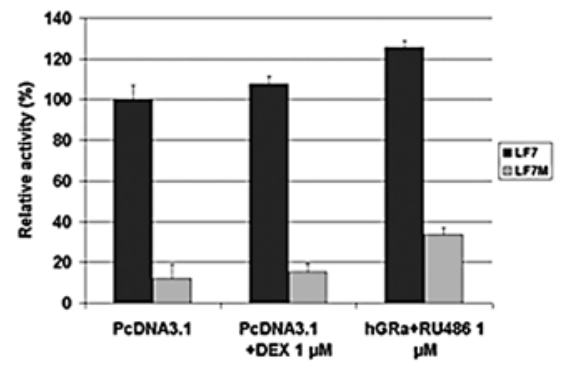

D

GR

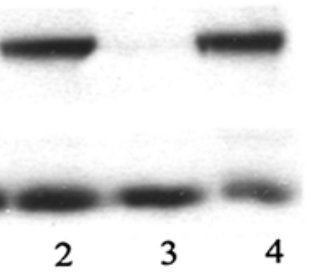

Figure 5. Existence of a functional GRE in the 125-bp region (-157 to -33) of 15-LOX-2 promoter. (A) Luciferase activities of LF4 downregulated by GR were reversed by specific GR inhibitors (siRNAs of GR and RU486) in LANCaP cells: Co-transfected with LF4 and parental plasmid pCDNA3 (activity normalized to 100); Co-transfected with LF4 and hGR $\alpha$ (60.73 \pm 2.09$)$; Co-transfected with LF4 and GRa plus siRNAs treatment (86.17 \pm 3.60$)$; Co-trasnfected with LF4 and hGR $\alpha$ plus RU486 treatment (172 \pm 7.78$)$. (B) Luciferase activities of LF7 downregulated by GR were reversed by specific GR inhibitors (siRNAs of GR and RU486) in LANCaP cells: Co-transfected with LF7 and parental plasmid pCDNA3 (activity normalized to 100). Co-transfected with LF7

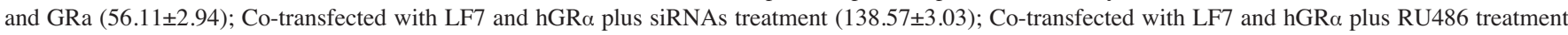
(290.18 \pm 9.79$)$. (C) Additional demonstration for this functional GRE through site-directed mutagenesis: LF7 (luciferase activity of LF7 normalized as 100) and LF7M (11.93 \pm 0.61$)$ without any treatment; LF7 (107.48 \pm 4.00$)$ and LF7M (15.23 \pm 1.018$)$ after DEX (1 $\mu$ M) treatment; LF7 (125.52 \pm 3.39$)$ and LF7M $(33.54 \pm 1.47)$ after RUM486 $(1 \mu \mathrm{M})$. (D) Western blot analysis of GR in prostate cancer cells LANCaP: Lane 1, LF4-pCDNA3.1; Lane 2, Co-transfection of LF4 and hGR $\alpha$; Lane 3, Co-tranfection of LF4-hGR $\alpha$ plus siRNAs treatment; Lane 4, Co-transfection of LF4-hGR $\alpha$ plus RU486 treatment.

To further examine whether or not the above GRE is functional, we performed site-directed mutagenesis to create a mutant GRE containing three mismatch mutations. This GRE mutant was called LF7M. This was generated using Change-IT Multiple Mutation Site Directed Mutagenesis kit following by the manufacturer's instruction. The mutations in the primer GRGSM-5 were the same as in GRGSM-4, which was used in EMSA (Table I). The luciferase activity of LF7M was found to dramatically decrease to $11.93 \pm 7.07$ after its normalization to 100 . The treatment with $\operatorname{DEX}(1 \mu \mathrm{M})$ did not result in significant increase in the luciferase activity of LF7M $(15.23 \pm 1.018)$, and the treatment with RUM486 $(1 \mu \mathrm{M})$ resulted in increase in the luciferase activity of LF7M (33.54 \pm 1.47 ), but not to the level of LF7 (Fig. 5C). Collectively, the above findings suggested the GRE exists and is functional in this 125 -bp key region $(-157$ to -33$)$ of the 15 -LOX-2 promoter.

\section{Discussion}

Through progressive dissection and analysis of $5^{\prime}$ untranslated structure of 15 -LOX-2 gene, we have determined that the 125-bp region (-157 to -33) in the LF4 fragment is critical for the promoter regulation of $15-\mathrm{LOX}-2$ gene in prostate cancer cells $\mathrm{LNCaP}$. This is because the luciferase activity $(111 \pm 6.48)$ of LF4 was similar (slightly reduced) to the dissected upstream fragments LF1-LF3, and activity of downstream fragment LF5 was dramatically reduced $(1.94 \pm 0.07)$ almost to the level of the control background. This was further demonstrated using the fragments LF6 and LF7 that share the same sequence with LF3 and LF4, but do not contain the 87-bp region downstream the transcription start site (TSS). This suggests that the sequence downstream of the TSS in 15LOX-2 promoter might not contain a special binding element(s) that significantly influences the gene expression in the prostate epithelial cancer cells LNCaP. Compared with the previous two studies on the promoter dissection and analysis for 15 -LOX-2 gene, we found this 125-bp region $(-157$ to -33$)$ is within the most active fragments identified in their studies $(5,9)$. In this study, we have advanced the 15LOX-2 promoter dissection into many smaller fragments and have determined the 125 -bp key region $(-157$ to -33$)$ for the promoter regulation of 15-LOX-2 gene. Therefore, we believe that the results from our study have advanced our research in revealing key transcriptional factors that regulate the 15-LOX-2 promoter in cancer cells.

Through the extensive computer analysis and powerful electrophoretic mobility shift assay (EMSA) (Fig. 3) and sitedirected mutagenesis (Fig. 5C), we have determined existence of functional GRE in the 125-bp key region of 15-LOX-2 gene. Furthermore, we have determined that dexamethasone (DEX), which is known to downregulate GR in prostate cancer 
cells (19), have reversed the inhibitory effect on the 15-LOX-2 promoter activity (Fig. 4). This discovery is very important in revealing the molecular mechanism on the tumor suppressing function of $15-\mathrm{LOX}-2$ gene. This is because glucocorticoid hormones are known to regulate proliferative, inflammatory and immune responses via its receptors GR (14-17). Clinically, glucocorticoid hormones, combined with or without chemotherapy, have been extensively used for the treatment of hormone refractory prostate cancer (HRPC). Glucocorticoids were even used as the 'standard' therapy arm in several randomized phase II-III clinical trials for the combination therapy of HRPC $(14,18,25)$. However, it still remains to be determined whether or not glucocorticoid hormones suppress prostate cancer growth through the activation of tumor suppressor gene $15-\mathrm{LOX}-2$ in vivo.

In addition, we have further confirmed the functional regulation of 15-LOX-2 promoter through the use of two specific GR inhibitors (siRNAs of GR and RU486) (Fig. 5). A siRNAs of GR is a short RNA sequence specific to GR that completely abolishes the expression of GR (21). In our study, we demonstrated that addition of this siRNA almost abolished expression of both endogenous and exogenous GR. RU486 is another well-known GR inhibitor, which specially binds glucocorticoid receptor, estrogen receptor and progesterone receptor and inhibits their functions, but does not affect their protein expression (24). In our study, we indeed see no significant change in the level of GR protein in the cells treated with RU486 (Fig. 5D). Of great interest is that the degree of inhibitory effect by RU486 is much greater than the siRNAs of GR. The reason for this difference is unknown. This may be due to inhibition of other proteins including estrogen receptor and progesterone receptor by RU486, which may be responsible for additional inhibitory effect by RU486.

The glucocorticoid receptor GR is a well-known transcription factor $(26,27)$. To our understanding, two major mechanisms on the gene regulation by GR: direct or indirect transcriptional regulation were previously reported $(28,29)$. The direct transcriptional regulation (transactivation) occurs via binding of the GR homodimer to palindromic promoter DNA sequences called glucocorticoid-response elements (GRE). The indirect regulation is mediated via cross-talk with other transcription factors (TFs) $(28,29)$. Most of such GRTF interactions repress the activity of partner TFs and their target genes (transrepression) (18). In this study, we have demonstrated an existence of a specific and functional GRE in the key region (-157 to -33) of 15-LOX-2 promoter (Fig. 3). However, it still remains unclear whether or not GR regulates 15-LOX-2 promoter through indirect transcriptional regulation mechanism. This certainly points to our future research direction.

GR expression was strongly decreased or absent in $70-85 \%$ of PC. Similar to primary prostate cancer, some prostate cancer cell lines including LNCaP, also lack GR expression (18). The GR expression is found to decrease at the highgrade prostatic intraepithelial neoplasm (HGPIN), early stage of prostatic carcinogenesis. The early loss of GR expression in prostate tumorigenesis resembles changes reported for estrogen receptor $\beta$, an inhibitor of prostate growth (30). In contrast, the expression of other steroid hormone receptors either remains stable, like AR, or is increased, like estrogen receptor $\alpha$ and progesterone receptor $(30,31)$. Remarkably, the loss of GR was specific only for the epithelial compartment of PCs. In benign and malignant prostate specimens alike, the stromal cells showed predominant nuclear localization of GR (32), suggesting an important role of GR specifically in prostate epithelium (18). 15-LOX-2 is normally expressed in apical epithelial cells of prostate epithelium, but either significantly reduced or lost in majority of prostate cancer (2). Decrease of 15-LOX-2 expression also starts at the HGPIN. In this study, we have demonstrated that the 15LOX-2 promoter can be negatively regulated by overexpression of GR in prostate cancer cells in vitro. However, we still do not know how 15-LOX-2 and GR interact in primary prostate cancer. Many questions still remain. For example, is 15 -LOX-2 inversely related to GR in terms of their expression pattern and function in an individual cell? Additional mechanistic studies are certainly required.

In summary, we have discovered that a 125-bp region (-157 to -33 ) of $15-\mathrm{LOX}-2$ promoter is critical for the promoter regulation of 15-LOX-2. In addition, we have demonstrated the existence of a specific and functional GR-responsive element (GRE) in this key region through computer analysis, electrophoretic mobility shift assay and site-directed mutagenesis. Overexpression of GR was shown to downregulate the promoter activity of 15-LOX-2 gene. This inhibitory effect was partially or completely reversed by steroid dexamethason (DEX) and specific GR inhibitors (siRNAs of GR and RU486). Results from our study have demonstrated that promoter activity of 15-LOX-2 was inhibited by overexpression of GR possibly through a direct binding mechanism.

\section{Acknowledgements}

We would like to thank Dr Julian R.E. Davis (Manchester University, UK) for pcDNA3hGR $\alpha$ and pcDNA3hGR 3 plasmids and Dr Fei Xiao (University of Stanford, CA) for the pGAW vector. The study described in this article is partially supported by the grants from Medical College of Wisconsin Cancer Center-American Cancer Society and from Radiation Therapeutic Oncology Group (RTOG).

\section{References}

1. Brash AR, Boeglin WE and Chang MS: Discovery of a second 15S-lipoxygenase in humans. Proc Natl Acad Sci USA 94: 6148-6152, 1997.

2. Shappell SB, Boeglin WE, Olson SJ, Kasper S and Brash AR: 15-lipoxygenase-2 (15-LOX-2) is expressed in benign prostatic epithelium and reduced in prostate adenocarcinoma. Am J Pathol 155: 235-245, 1999.

3. Xu XC, Shappell SB, Liang Z, et al: Reduced 15S-lipoxygenase- 2 expression in esophageal cancer specimens and cells and upregulation in vitro by the cyclooxygenase- 2 inhibitor, NS398. Neoplasia 5: 121-127, 2003.

4. Wang D, Chen S, Feng Y, et al: Reduced expression of 15lipoxygenase 2 in human head and neck carcinomas. Tumour Biol 27: 261-273, 2006.

5. Subbarayan V, Xu XC, Kim J, et al: Inverse relationship between 15-lipoxygenase-2 and PPAR-gamma gene expression in normal epithelia compared with tumor epithelia. Neoplasia 7 : 280-293, 2005.

6. Jack GS, Brash AR, Olson SJ, et al: Reduced 15-lipoxygenase-2 immunostaining in prostate adenocarcinoma: correlation with grade and expression in high-grade prostatic intraepithelial neoplasia. Hum Pathol 31: 1146-1154, 2000 
7. Tang S, Bhatia B, Maldonado CJ, et al: Evidence that arachidonate 15-lipoxygenase 2 is a negative cell cycle regulator in normal prostate epithelial cells. J Biol Chem 277: 16189-16201, 2002.

8. Bhatia B, Tang S, Yang P, et al: Cell-autonomous induction of functional tumor suppressor 15-lipoxygenase 2 (15-LOX2) contributes to replicative senescence of human prostate progenitor cells. Oncogene 24: 3583-3595, 2005.

9. Tang S, Bhatia B, Zhou J, et al: Evidence that Sp1 positively and Sp3 negatively regulate and androgen does not directly regulate functional tumor suppressor 15-lipoxygenase 2 (15LOX2) gene expression in normal human prostate epithelial cells. Oncogene 23: 6942-6953, 2004.

10. Krieg P, Marks F and Furstenberger G: A gene cluster encoding human epidermis-type lipoxygenases at chromosome 17p13.1: cloning, physical mapping, and expression. Genomics 73: 323-330, 2001

11. Ham J, Thomson A, Needham M, Webb P and Parker M: Characterization of response elements for androgens, glucocorticoids and progestins in mouse mammary tumour virus. Nucleic Acids Res 16: 5263-5276, 1988.

12. Beato M: Gene regulation by steroid hormones. Cell 56: 335-344, 1989.

13. Beato M, Chalepakis G, Schauer M and Slater EP: DNA regulatory elements for steroid hormones. J Steroid Biochem 32: 737-747, 1989.

14. Fakih M, Johnson CS and Trump DL: Glucocorticoids and treatment of prostate cancer: a preclinical and clinical review. Urology 60: 553-561, 2002.

15. Li Z, Chen Y, Cao D, et al: Glucocorticoid up-regulates transforming growth factor-beta (TGF-beta) type II receptor and enhances TGF-beta signaling in human prostate cancer PC-3 cells. Endocrinology 147: 5259-5267, 2006.

16. Yano A, Fujii Y, Iwai A, Kageyama Y and Kihara K: Glucocorticoids suppress tumor angiogenesis and in vivo growth of prostate cancer cells. Clin Cancer Res 12: 3003-3009, 2006.

17. Gao QZ, Lu JJ, Liu ZD, Zhang H, Wang SM and Xu H: Dexamethasone suppresses DU145 cell proliferation and cell cycle through inhibition of the extracellular signal-regulated kinase 1/2 pathway and cyclin D1 expression. Asian J Androl 10: 635-641, 2008

18. Yemelyanov A, Czwornog J, Chebotaev D, et al: Tumor suppressor activity of glucocorticoid receptor in the prostate. Oncogene 26: 1885-1896, 2007.

19. Nishimura K, Nonomura N, Satoh E, et al: Potential mechanism for the effects of dexamethasone on growth of androgenindependent prostate cancer. J Natl Cancer Inst 93: 1739-1746, 2001.
20. Koufali MM, Moutsatsou P, Sekeris CE and Breen KC: The dynamic localization of the glucocorticoid receptor in rat C6 glioma cell mitochondria. Mol Cell Endocrinol 209: 51-60, 2003.

21. Felinski EA, Cox AE, Phillips BE and Antonetti DA: Glucocorticoids induce transactivation of tight junction genes occludin and claudin-5 in retinal endothelial cells via a novel cis-element. Exp Eye Res 86: 867-878, 2008.

22. Dondi D, Maggi R, Scaccianoce E, Martini L, Motta M and Poletti A: Expression and role of functional glucocorticoid receptors in the human androgen-independent prostate cancer cell line, DU145. J Mol Endocrinol 26: 185-191, 2001.

23. Onica T, Nichols K, Larin M, et al: Dexamethasone-mediated up-regulation of human CYP2A6 involves the glucocorticoid receptor and increased binding of hepatic nuclear factor 4 alpha to the proximal promoter. Mol Pharmacol 73: 451-460, 2008.

24. Martindale RJ: The extra pharmacopoeia. 31st edition, UK: Royal Pharmaceutical Society, London, 1996.

25. Koutsilieris M, Mitsiades C, Dimopoulos T, et al: Combination of dexamethasone and a somatostatin analogue in the treatment of advanced prostate cancer. Expert Opin Investig Drugs 11: 283-293, 2002.

26. Beato M, Herrlich P and Schutz G: Steroid hormone receptors: many actors in search of a plot. Cell 83: 851-857, 1995.

27. Chebotaev D, Yemelyanov A, Zhu L, Lavker RM and Budunova I: The tumor suppressor effect of the glucocorticoid receptor in skin is mediated via its effect on follicular epithelial stem cells. Oncogene 26: 3060-3068, 2007.

28. De Bosscher K, Vanden Berghe W and Haegeman G: The interplay between the glucocorticoid receptor and nuclear factorkappaB or activator protein-1: molecular mechanisms for gene repression. Endocr Rev 24: 488-522, 2003.

29. Necela BM and Cidlowski JA: Mechanisms of glucocorticoid receptor action in noninflammatory and inflammatory cells. Proc Am Thorac Soc 1: 239-246, 2004.

30. Fixemer T, Remberger K and Bonkhoff H: Differential expression of the estrogen receptor beta (ERbeta) in human prostate tissue, premalignant changes, and in primary, metastatic, and recurrent prostatic adenocarcinoma. Prostate 54: 79-87, 2003.

31. Torlakovic E, Lilleby W, Berner A, et al: Differential expression of steroid receptors in prostate tissues before and after radiation therapy for prostatic adenocarcinoma. Int J Cancer 117: 381-386, 2005.

32. Mohler JL, Chen Y, Hamil K, et al: Androgen and glucocorticoid receptors in the stroma and epithelium of prostatic hyperplasia and carcinoma. Clin Cancer Res 2: 889-895, 1996. 